\title{
O EFEITO DA REALIDADE VIRTUAL NA CAPACIDADE FUNCIONAL DE MEMBROS SUPERIORES EM INDIVÍDUOS COM HEMIPARESIA
}

Nicoly Ribeiro Uliam, Isabella Cristina Leoci, Silas de Oliveira Damasceno, Caroline Nunes Gonzaga, Isabela Bortolim Frasson, Alice Haniuda Moliterno, Guilherme Yassuyuki Tacao, Lúcia Martins Barbatto, Isabella Menezes Silva, Paola Larissa de Araujo Biazini, Augusto Cesinando de Carvalho

Universidade Estadual Paulista - UNESP. Presidente Prudente, SP. e-mail: nicoly.ruliam@gmail.com

\section{RESUMO}

O acidente vascular encefálico (AVE) pode acarretar a perda total e/ou parcial da função de um dos membros superiores, sendo sua reabilitação um dos focos principais dos fisioterapeutas. $O$ objetivo foi analisar os efeitos da realidade virtual na capacidade funcional dos membros superiores em indivíduos com hemiparesia. Inicialmente foram avaliados quanto a destreza manual pelo Nine Hole Peg Test (NHPT) e Teste de Caixa de Blocos (TCB) para, posteriormente serem direcionados a realização de um protocolo de 16 sessões utilizando a realidade virtual, por meio de jogos do console Nintendo Wii ${ }^{\mathrm{TM}}$. Foram incluídos 10 participantes no estudo com idade média de $64,5 \pm 9,54$ não tendo demonstrado resultados significativos quando comparado os momentos, apenas um pequeno efeito $(d=0,23)$ foi encontrado em membro superior esquerdo no NHPT. Conclui-se então que não houve melhora significativa na capacidade funcional de membros superiores utilizando realidade virtual em indivíduos com hemiparesia.

Palavras-chave: extremidade superior, acidente vascular cerebral, fisioterapia, realidade virtual, neurologia.

\section{THE EFFECT OF VIRTUAL REALITY-BASED TREATMENT ON UPPER LIMB FUNCTIONAL CAPACITY IN INDIVIDUALS WITH HEMIPARESIS}

\begin{abstract}
Stroke may lead to total and/or partial loss of normal function in one of the upper limbs, and the rehabilitation is one of the main focuses of physiotherapists. The objective was to analyze the effects of virtual reality on upper limb functional capacity in individuals with hemiparesis. Initially they were evaluated for manual dexterity by the Nine Hole Peg Test (NHPT) and Box and Blocks Test (BBT) then be conducted to perform a 16-session protocol using virtual reality game through Nintendo $\mathrm{Wii}^{\mathrm{TM}}$ console. $^{\mathrm{TM}}$. We included 10 individuals with mean age of $64.5 \pm 9.54$ and did not demonstrated significant results when comparing the moments, only a small effect $(d=0.23)$ was found in the left upper limb in the NHPT. It was concluded, there was no significant improvement in the functional capacity of the upper limbs using virtual reality in individuals with hemiparesis.
\end{abstract}

Keywords: Upper extremity, stroke, physical therapy, virtual reality, neurology.

\section{INTRODUÇÃO}

O acidente vascular encefálico (AVE) é descrito como a perda súbita da função neurológica, podendo ser ele causado por um evento isquêmico ou hemorrágico ${ }^{1}$. Apesar de ter apresentado uma diminuição na incidência e mortalidade dentro dos últimos anos ${ }^{2,3}$, ainda é considerado como uma das principais causas de 
mortalidade e incapacidade, dentro do contexto mundial ${ }^{4}$, sendo considerado como a epidemia do século XXI pela Organização Mundial de Saúde $(\mathrm{OMS})^{5}$.

Como principais fatores de riscos para o AVE temos: hipertensão, hiperlipidemia, diabetes mellitus, tabagismo e terapia antitrombótico ${ }^{2}$, dos quais são o foco para sua prevenção. Diante deste cenário a mudança de estilo de vida tem sido adotada como principal meio de prevenir esse problema.

Dentre os acometimentos do AVE temos como principal a hemiplegia/hemiparesia, que se caracterizam por uma deficiência motora em um lado do corpo ${ }^{6}$. As alterações características são sensoriais, cognitivas e motoras, e consequentemente, diminuição da capacidade de deambulação, de realizar as atividades de autocuidados e de vida diária, e participação comunitária ${ }^{7,8}$. Mostra-se diante desses acometimentos a necessidade de um atendimento multidisciplinar, desde $\mathrm{O}$ acometimento, fase aguda, até na fase crônica ${ }^{1,7,9}$.

A reabilitação física se mostra efetiva diante da recuperação da função desses indivíduos, e dentro dessa reabilitação existem diversas terapias e métodos que buscam de forma geral a melhora do paciente e sua independência ${ }^{10}$. Dentre os objetivos e focos da fisioterapia temos a restauração do controle motor nas atividades físicas de forma geral, melhora da função de membro superior, melhora da inserção social e também instruir e preparar o paciente para lidar com as atividades de vida diária $^{11}$. Dentre essas terapias temos a realidade virtual (RV) que tem se mostrado atualmente uma ferramenta importante e centrada nas características dos pacientes ${ }^{12}$.

A RV é uma tecnologia que permite que os pacientes experimentem e interajam com ambientes diversos e tridimensionais, por meio de jogos, que permitem um feedback e um controle de intensidade, permitindo assim 0 controle do treinamento ${ }^{13}$. Crosbie et al. ${ }^{13}$, traz em seu estudo que esse meio de terapia se mostrou segura e aceitável para os pacientes que tiveram um AVE, porém necessário novos estudos para se garantir sua eficácia.

A maior evolução do tratamento de reabilitação de indivíduos que sofreram um AVE se encontra nos três primeiros meses pós ocorrido, porém a recuperação continua evidente mesmo após esse período ${ }^{9}$. Em relação aos membros superiores, um dos braços está acometidos em cerca de mais de dois terços dos pacientes admitidos com AVE nos hospitais, apresentando uma hemiparesia e consequentemente, função reduzida e limitada das mãos ${ }^{9}$.

Diante desse cenário, o objetivo desse trabalho foi analisar os efeitos de um protocolo de intervenção utilizando a realidade virtual, na capacidade funcional dos membros superiores de indivíduos com hemiparesia, em fase crônica.

\section{METODOLOGIA}

Os participantes foram recrutados num centro de atendimento de Fisioterapia e Reabilitação de uma universidade em Presidente Prudente. Os procedimentos adotados nesta pesquisa obedecem aos Critérios da Ética em Pesquisa com Seres Humanos conforme resolução no. 466/2012 do Conselho Nacional de Saúde. Nenhum dos procedimentos usados oferece riscos à dignidade dos integrantes.

Trata-se de um estudo clínico experimental, longitudinal, prospectivo e de braço único. $O$ estudo foi aprovado pelo Comitê de Ética em pesquisa da Faculdade de Ciências e Tecnologia de Presidente Prudente - FCT/UNESP (CAAE:90293618.5.0000.5402).

Como critérios de inclusão foram utilizados: indivíduos com hemiparesia unilateral com encaminhamento médico e tempo de lesão $\geq$ há 12 meses; capacidade de realizar a marcha com ou sem auxílio de órtese; que apresentasse fraqueza muscular do membro superior acometido identificadas pela dinamometria dos flexores dos dedos e punho (diferença entre o lado acometido e não acometido); ausência de déficits cognitivos avaliados pelo Mini-exame do Estado Mental (ponto de corte para indivíduos analfabetos 18/19 e para indivíduos com instrução escolar 24/25) ${ }^{14}$.

Como critérios de exclusão foram utilizados: indivíduos com dupla hemiparesia; tempo de lesão inferior a 12 meses; afasia sensitiva; outras condições de saúde adversas, como outras doenças neurológicas ou ortopédicas não relacionadas ao AVE.

Inicialmente foi realizada uma entrevista individual com as integrantes para coleta de dados e verificação dos critérios de inclusão. Após serem aceitos pelos critérios de inclusão e aceitarem participar da pesquisa foram esclarecidas todas as dúvidas sobre o protocolo e 
assinaram o termo de consentimento livre esclarecido (TCLE), passando a integrar a pesquisa. Em seguida, passaram pela avaliação dos testes funcionais específicos (AV1), sendo eles o Nine hole peg test (NHPT) e o Teste de caixa de blocos (TCB).

O NHPT é um teste responsável por avaliar a coordenação motora fina dos membros superiores, por meio do desempenho cronometrado de colocar e retirar nove pinos dos orifícios de um bloco de madeira ${ }^{15,16}$. A posição inicial do instrumento é sobre uma mesa, centralizado imediatamente a frente do indivíduo, e este recebeu a orientação para que retire todos os pinos inicialmente e em seguida recoloque-os de volta no bloco. $O$ teste se iniciou pelo lado não acometido, e após, faz-se com o lado acometido, sendo computado os segundos necessários para realização. Foi realizado uma breve demonstração antes de o participante iniciar o teste ${ }^{17 .}$

O TCB é uma forma simples e rápida de avaliar e mensurar a destreza manual grossa de indivíduos com hemiparesia, onde permite observar a qualidade da função manual e mensurar a velocidade de manipulação de objetos, além de ser um instrumento de fácil aplicação e confecção ${ }^{15}$. Para a realização do teste é necessário um cronômetro, uma caixa de madeira com uma divisória ao meio e 150 blocos de madeira em formato cúbico. $O$ instrumento fica posicionado na horizontal, em uma mesa logo a frente do indivíduo que se posiciona sentado de maneira confortável em uma cadeira.

Inicialmente os blocos ficaram localizados em um dos lados da caixa, e então o participante recebeu a orientação de transferir um bloco por vez para o outro lado, o mais rápido possível, sendo cronometrado um minuto. O teste foi iniciado com o membro superior não acometido, e em seguida com o acometido. Antes de iniciar foi realizado um treino com duração de 15 segundos. O teste é mensurado pela quantidade de blocos que o indivíduo consegue passar de um lado para o outro dentro de um minuto, sendo pontuado um ponto para cada bloco ${ }^{18,19}$.

Após finalizadas as avaliações os participantes foram encaminhados para o tratamento que consistiu em um protocolo com realidade virtual utilizando o console Nintendo $\mathrm{Wii}^{\mathrm{TM}}$, no qual foram utilizados o jogo Wii Fit TM Plus e jogo Wii Sports Resort TM com o acessório Wii Balance Board (plataforma) e o controle. Os integrantes da pesquisa, antes de iniciarem o tratamento, passaram por uma sessão de familiarização onde foram orientados sobre o funcionamento do aparelho e de cada jogo.

Durante a realização das sessões o participante foi posicionado em pé em frente a uma televisão com distância de 2 metros, entre eles. Foram realizados seis jogos descritos na Tabela 1, incluindo equilíbrio, coordenação e destreza. O protocolo consistiu em duas sessões por semana durante oito semanas, totalizando 16 sessões, tendo 45 minutos de duração cada. Ao final do protocolo foi realizada uma nova avaliação (AV2) utilizando os mesmos testes da avaliação inicial (AV1).

Os dados foram tabulados em planilhas pré-definidas no software Microsoft Office Excel e testados quanto à distribuição normal pelo teste de Shapiro wilk. As estatísticas descritivas (média e desvio padrão) foram operacionalizadas pelo Statistical Software for Social Sciences (SPSS Inc. Chicago, IL) versão 18.0, bem como, para comparar resultados das escalas será utilizado o teste T-student para amostras pareadas ou teste de Wilcoxon, considerando significante o valor de $p<0,05$. 
Tabela 1. Descrição dos jogos do Nintendo Wii.

\begin{tabular}{|c|c|}
\hline JOGOS & DESCRIÇÃO \\
\hline Free Run & $\begin{array}{l}\text { Esse jogo é caracterizado como uma caminhada no lugar em velocidade constante, } \\
\text { utilizado para aquecimento durante cinco minutos. }\end{array}$ \\
\hline $\begin{array}{l}\text { Hula } \\
\text { Hoop }\end{array}$ & $\begin{array}{l}\text { Consiste em um jogo que o indivíduo simula um bambolê, com movimentos circulares de } \\
\text { quadril sobre a plataforma Wii. O objetivo é manter os bambolês girando em torno do } \\
\text { quadril sem deixá-los cair. }\end{array}$ \\
\hline Perfect 10 & $\begin{array}{l}\text { O objetivo do jogo é realizar movimentos com o quadril (para frente, para trás e para os } \\
\text { lados) sobre a plataforma Wii Balance Board ativando os cogumelos coloridos enumerados } \\
\text { de maneira com que se some } 10 \text { pontos. }\end{array}$ \\
\hline $\begin{array}{l}\text { Penguin } \\
\text { Slide }\end{array}$ & $\begin{array}{l}\text { Neste jogo o indivíduo é representado por um pinguim em uma plataforma de gelo sobre } \\
\text { água. É realizado utilizando a plataforma Wii Balance Board. O objetivo é realizar descarga } \\
\text { de peso nos membros inferiores a fim de inclinar a plataforma de gelo e se alimentar do } \\
\text { maior número de peixes possíveis. }\end{array}$ \\
\hline Free Step & $\begin{array}{l}\text { O objetivo deste jogo é subir e descer sobre a plataforma (Wii Balance Board) mantendo o } \\
\text { ritmo e alternando os pés de minuto em minuto. }\end{array}$ \\
\hline $\begin{array}{l}\text { Table } \\
\text { Tennis }\end{array}$ & $\begin{array}{l}\text { Consiste em um jogo que simula o tênis de mesa, no qual o indivíduo realiza movimentos } \\
\text { de flexão de cotovelo com o controle do Nintendo Wii nas mãos. }\end{array}$ \\
\hline
\end{tabular}

\section{RESULTADOS}

No presente estudo foram avaliados no total 10 indivíduos, sendo cinco deles do gênero feminino e cinco masculino, com hemiparesia unilateral dos quais dois apresentavam comprometimento do hemicorpo direito e oito do hemicorpo esquerdo. Entre os participantes podemos encontrar as seguintes características de idade e escolaridade descritas na Tabela 2.

A Tabela 3 mostra os dados descritivos coletados na AV1 de ambos os testes.

A Tabela 4 mostra os dados descritivos de valores coletados na AV2, de ambos os testes, dos indivíduos que realizaram o protocolo de intervenção.

Os resultados encontrados comparando AV1 e AV2 do TCB não foi significativo para dados normais e pareados $(p=0,37)$ e também não foi significativo para dados não-normais e pareados $(p=0,798)$. No teste NHPT também não foi encontrado nenhum resultado significativo comparando o antes e pós-intervenção (dados normais e pareados $p=0,36$; dados não normais $e$ pareados $p=0,72$ ).
Foi aplicado também o Effect Size descrito na Tabela 5, porém foi encontrado apenas um pequeno efeito no nine hole peg test no membro superior direito.

Tabela 2. Dados sociodemográficos. Média, desvio padrão, mínimo e máximo.

\begin{tabular}{lc}
\hline Variáveis & Participantes \\
\hline Idade (anos) & $64,5 \pm 9,54$ \\
& {$[47-78]$} \\
Escolaridade (anos) & $8,3 \pm 4,06$ \\
& {$[1-16]$} \\
\hline
\end{tabular}

Tabela 3. Valores médios e dos desvios padrão.

\begin{tabular}{lc}
\hline Variáveis (AV1) & Participantes \\
\hline TCB (MSD) & $48,6 \pm 7,18$ \\
TCB (MSE) & $33,8 \pm 15,54$ \\
NHPT (MSD) & $26,94 \pm 10,15$ \\
NHPT (MSE) & $32,46 \pm 22,71$
\end{tabular}

MSD = Membro superior direito; $\mathrm{MSE}=$ Membro superior esquerdo. 
Tabela 4. Valores médios e dos desvios padrão.

\begin{tabular}{cc}
\hline Variáveis (AV2) & Participantes \\
\hline TCB (MSD) & $49,2 \pm 7,97$ \\
TCB (MSE) & $35,5 \pm 16,57$ \\
NHPT (MSD) & $25,14 \pm 5,87$ \\
NHPT (MSE) & $30,46 \pm 22,94$
\end{tabular}

MSD = Membro superior direito; $\mathrm{MSE}=$ Membro superior esquerdo.
Tabela 5. Valores de Effect Size para o teste caixa de blocos e nine hole peg test.

\begin{tabular}{lcc}
\hline Variáveis & $\begin{array}{c}\text { Effect Size } \\
\text { (d) }\end{array}$ & Classificação \\
\hline TCB (MSD) & 0,08 & Efeito insignificante \\
TCB (MSE) & 0,11 & Efeito insignificante \\
NHPT (MSD) & 0,23 & Pequeno efeito \\
NHPT (MSE) & 0,09 & Efeito insignificante
\end{tabular}

MSD = Membro superior direito; $\mathrm{MSE}=$ Membro superior esquerdo.

crônicos, utilizando um treinamento funcional especifico e intensivo de RV, porém estudos que avaliam de forma mais completa o potencial desse treinamento, ainda se encontram em andamento. Já Yates et al. ${ }^{22}$, ressalta que a terapia com RV se demonstra mais eficaz na reabilitação durante a fase aguda do AVE, e relata também que há certa dificuldade em se encontrar estudos que apresentem resultados significantes, que demonstrem a eficácia da RV na melhora funcional de extremidades superiores.

O uso da RV em ambiente de reabilitação clínica ainda é pouco frequente, apesar das pesquisas estarem cada vez mais prevalentes. 0 que inicialmente foi criado apenas como forma de recreação, está sendo atualmente de maneira intensa, adaptado para fins terapêuticos e de reabilitação nos mais diversos âmbitos da saúde $^{23}$. O efeito não significante do atual estudo pode estar relacionado a essa dificuldade de adaptação, ou seja, apesar do número crescente de estudos, ainda são encontradas dificuldades no momento de estabelecer um protocolo para determinados objetivos utilizando essa tecnologia.

Com isso são necessários cada vez mais estudos que demonstrem a eficácia dessas adaptações, afim de se definir futuramente protocolos eficazes na melhoria dessa população, utilizando a tecnologia como uma ferramenta importante na melhora das capacidades funcionais dos pacientes. A partir disso, possibilitar e fortalecer a aplicabilidade clínica da RV.

Uma das limitações do presente estudo, foi que este buscou desenvolver um protocolo com uma abordagem global do paciente, afim de estudo um resultado positivo em indivíduos 
despertar interesse e habilidades do paciente, estimulando-o dentro da reabilitação, porém uma conduta mais direcionada aos membros superiores poderia demonstrar melhores efeitos dentro do âmbito da reabilitação funcional dessa região do corpo. Outra limitação foi o número de participantes do estudo.

Concluímos então, que não houve diferença significativa no uso desse protocolo de RV na melhora das capacidades funcionais de membros superiores na população investigada. Porém a RV tem se mostrado eficaz em muitos outros estudos, com isso, é recomendado que sejam feitos novos estudos com uma população maior com a utilização de outros jogos, ou até mesmo outros meios de tecnologias que proporcionem a RV, afim de determinar um protocolo de RV eficaz na melhora da função de membros superiores em indivíduos com hemiparesia crônica.

\section{CONFLITO DE INTERESSE}

Os autores declaram não haver qualquer potencial conflito de interesse que possa interferir na imparcialidade deste trabalho científico.

\section{REFERÊNCIAS}

1. Langhorne P, Collier JM, Bate PJ, Thuy MNT, Bernhardt J. Very early versus delayed mobilisation after stroke. Cochrane Database Syst Rev. 2018;10: Art. N. CD006187. DOI: http://dx.doi.org/10.1002/14651858.CD006187.p $\underline{\mathrm{ub3}}$

2. Guzik A, Bushnell C. Stroke epidemiology and risk fator management. Continuum. 2017;23(1):15-39. DOI: http://dx.doi.org/10.1212/CON.00000000000004 $\underline{16}$

3. Bao X, Mao Y, Lin Q, Qiu Y, Chen S, Li L et al. Mechanism of Kinect-based virtual reality training for motor functional recovery of upper limbs after subacute stroke. Neural Regen Res. 2013;8(31):2904-13.

DOI:

http://dx.doi.org/10.3969/j.issn.1673-

5374.2013.31.003

4. Laver KE, George S, Thomas S, Deutsch JE, Crotty M. Virtual reality for stroke rehabilitation. Cochrane Database Syst Rev. 2011;9: Art. N.
CD008349.

DOI: http://dx.doi.org/10.1002/14651858.CD008349.p $\underline{\mathrm{ub2}}$

5. Sarikaya H, Ferro J, Arnold M. Stroke Prevention - Medical and Lifestyle Measures. Eur Neurol. 2015;73:150-7.

DOI:

https://doi.org/10.1159/000367652

6. Fonseca ER. Efeito terapêutico da realidade virtual em hemiplégicos após Acidente Vascular Cerebral: Ensaio clínico randomizado [Dissertação]. Salvador: Escola Bahiana de Medicina e Saúde Pública, 2015.

7. Mehrholz J, Thomas S, Elsner B. Treadmill training and body weight support for walking after stroke. Cochrane Database Syst Rev. 2014;1: Art. N. CD002840. DOI: https://doi.org/10.1002/14651858.CD002840.pu $\underline{\mathrm{b} 3}$

8. Laver KE, George S, Thomas S, Deutsch JE, Crotty M. Virtual reality for stroke rehabilitation. Cochrane Database Syst Rev. 2011;9: Art. N. CD008349.

DOI: http://dx.doi.org/10.1002/14651858.CD008349.p $\underline{\mathrm{ub2}}$

9. Mehrholz J, Pohl M, Platz T, Kugler J, Elsner B. Electromechanical and robot-assisted arm training for improving activitiesof daily living, arm function, and arm muscle strength after stroke. Cochrane Database Syst Rev. 2018;9: Art. N. CD006876.

DOI: http://dx.doi.org/10.1002/14651858.CD006876.p $\underline{\mathrm{ub} 5}$

10. Pollock A, Baer G, Campbell P, Choo PL, Forster A, Morris J, Pomeroy VM, Langhorne P. Physical rehabilitation approaches for the recovery of function and mobility following stroke. Cochrane Database Syst Rev. 2014:4: Art. N. CD001920. DOI: http://dx.doi.org/10.1002/14651858.CD001920.p $\underline{\text { ub3 }}$

11. Van Peppen RPS, Kwakkel G, WoodDauphinee S, Hendriks HJM, Van der Wees PhJ, Dekker J. The impact of physical therapy on functional outcomes after stroke: what's the evidence? Clin Rehab. 2004;18:833-62. DOI: https://doi.org/10.1191/0269215504cr843oa 
12. Palma GC, Freitas TB, Bonuzzi GM, Soares MA, Leite PH, Mazzini NA, Almeida MR, Pompeu JE, Torriani-Pasin C. Effects of virtual reality for stroke individuals based on the International Classification of Functioning and Health: a systematic review. Topics Stroke Rehab. 2017;24(4):269-78. DOI: http://dx.doi.org/10.1080/10749357.2016.12503 $\underline{73}$.

13. Crosbie JH, Lennon S, McGoldrick MC, McNeill $\mathrm{MD}, \mathrm{McDonough} \mathrm{SM}$. Virtual reality in the rehabilitation of the arm after hemiplegic stroke: a randomized controlled pilot study. Clin Rehabil. 2012;26(9):798-806. DOI: http://dx.doi.org/10.1177/0269215511434575

14. Bertolucci PHF, Brucki SMD, Campacci SR, Juliano Y. O mini-exame do estado mental em uma população geral. Arq Neuropsiquiatr. 1994, 52(1):1-7. DOI: https://doi.org/10.1590/50004$\underline{282 \times 1994000100001}$

15. Beebe JA, Lang CE. Relationships and responsiveness of six upper extremity function tests during the first 6 months of recovery after stroke. J Neurol Phys Ther. 2009;33(2):96-103. DOI:

https://doi.org/10.1097/NPT.0b013e3181a33638

16. Mathiowetz V, Weber K, Kashman N, Volland G. Adult norms for the Nine Hole Peg Test of finger dexterity. Occup Ther J Res. 1985;5(1):2438.

DOI: https://doi.org/10.1177/153944928500500102

17. Grice KO, Vogel KA, Le V, Mitchell A, Muniz S, Vollmer MA. Adult norms for a commercially available nine hole peg test for finger dexterity. Am J Occup Ther. 2003;57(5):570-3. DOI: https://doi.org/10.5014/ajot.57.5.570

18. Guimarães R, Blascovi-Assis SM. Uso do teste caixa e blocos na avaliação de destreza manual em crianças e jovens com síndrome de Down. Rev Ter Ocup. 2012;23(1):98-106. DOI: http://dx.doi.org/10.11606/issn.22386149.v23i1p $\underline{98-106}$

19. Mathiowetz V, Volland G, Kashman N, Weber K. Adult norms for the Box and Block Test of manual dexterity. Am J Occup Ther.
1985;39(6):386-91.

DOI:

https://doi.org/10.5014/ajot.39.6.386

20. Wang Z, Wang P, Xing L, Mei L, Zhao J, Zhang $T$. Leap motion-based virtual reality training for improving motor functional recovery of upper limbs and neural reorganization in subacute stroke patients. Neural Regen Res. 2017;12(11):1823-31.

DOI:

https://doi.org/10.4103/1673-5374.219043

21. Perez-Marcos D, Chevalley O, Schmidlin T, Garipelli G, Serino A, Vuadens $P$ et al. Increasing upper limb training intensity in chronic stroke using embodied virtual reality: a pilot study. J Neuroeng Rehabil. 2017; 14: 119. DOI: https://doi.org/10.1186/s12984-017-0328-9

22. Yates $M$, Kelemen A, Lanyi CS. Virtual reality gaming in the rehabilitation of the upper extremities post-stroke. Brain Injury. 2016;30(7):855-63. DOI: http://dx.doi.org/10.3109/02699052.2016.11441 $\underline{46}$

23. Laver KE, Lange B, George S, Deutsch J, Saposnik G, Crotty M. Virtual reality for stroke rehabilitation. Cochrane Database Syst Rev. 2017;11: Art. N. CD008349. DOI: https://doi.org/10.1002/14651858.CD008349.pu b4 